\title{
A Robotic Platform For Endovascular Aneurysm Repair
}

\author{
Iris Naudin and Minh Tu Pham and Richard Moreau and Arnaud Lelevé
}

\begin{abstract}
An EndoVascular Aneurysm Repair (EVAR) is a procedure used to fix an aneurysm of the aorta. In this procedure, a guide is inserted by the femoral artery. This guide goes through to the height of the aneurysm and then a catheter follows the guide. Next, a stent graft is deployed in order to repair the aortic aneurysm. The objectives of our work is to develop a low-cost robotic system and implement a program that helps the trajectory planning during an endovascular operation. More precisely, this program can predict if the aorta will break or not depending on the guide used. Such a robotic platform could serve as a teaching instrument by creating an environment for young surgeons in which they will be able to practice their skills to perform an EVAR. This paper describes the different components of this platform and provides some experimental results.
\end{abstract}

\section{INTRODUCTION}

The need of advanced technology in the endovascular field has attracted many research studies. As a result we can find an extensive number of improvements and new ideas with the goals of reducing time, facilitate surgery and improve the outcome of the operation [7]. [9] is an impressive work which consists of the develpment of a robot for a vascular interventional surgery (VIS) that has a masterslave structure and involves a fluoroscopic guidance. When one compares conventional surgery to robotic surgery it is common to find out that it takes a little bit more time for the robotic one to perform the surgery [2]. This can be due to the lack of experience in the surgeon to use the robot itself. Furthermore, it minimizes surgeon-based error. However, VIS can affect the surgeon with radiation sickness and injuries to skin and eyes. This is one of the reasons why medical robots were designed. [2] presents several robots with different assets: Firstly, it deals with the NaviCath system which includes a bedside unit and an operator control unit. It is controlled by a touch screen-controlled console and a joystick. Secondly, it mentions the Sensei robotic catheter control system (CCS) which facilitates not only the control but also the accurate positioning of a catheter within the cardiovascular system. Furthermore, it shows a catheter operating robot system (CORS) for vascular neurosurgery which the surgeon manipulates the robot away from the site where the surgery is made. Finally a remotecontrol VIS robot dedicated to cardiovascular interventions is developed. It has three outstanding improvements to the systems mentioned above: Its mechanism has a supporting manipulator and a catheter navigator, the image navigation systems takes the model from the $2 \mathrm{D}$ projection image into a

All the authors are with Laboratoire Ampère, UMR CNRS 5005, Universiteé de Lyon, INSA Lyon, F69621, Villeurbanne, France name.surnamedinsa-lyon.fr
3D vascular model, a novel piezoelectric sensor to measure the contact force between the catheter and the arterial wall is presented. The advantages of such a system is i) to avoid Xray radiations of the surgeon by the provision of a convenient working environment, ii) to reduce a surgical risk due to artificial reasons by quantifying the catheter motion, iii) to improve surgical safety by the measurement of the contact force between the arterial wall and the catheter tip. In [8] the authors mention the use of position electromagnetic tracking system to localized a sensor attached to the catheter in combination of CT angiography. This method can produce high quality 3D images with blood vessels clearly presented, which will serve as the anatomical atlas during the surgical navigation. The imagery is created by two virtual cameras that are placed in the same direction of the two sensors and provide feedback to the surgeon in order to know what he/she is doing at the moment of placing the catheter in the targeted vessel.

The present study aims to develop a low cost robot which will help the planning of an endovascular operation and could also be implemented in education using the same instruments that a normal EVAR operation. This robot will act as a simulator of an endovascular surgery to repair an abdominal aortic aneurysm, converting it into a great asset for education. It is important to understand that for education purposes it is not necessary to have an exact replica of the operation, however there are important elements that are crucial for the knowledge we want surgeons to acquire. One of them is a haptic feedback. Haptics refers to a set of technological devices that interact with humans through the sense of touch, in other words, it is any form of nonverbal communication involving touch [3]. This feedback will help the surgeons to recognize the forces applied on the aorta. The advantages of this method in comparison with other methods such as optical based, acoustic based and x-ray based are that it is not time consuming; it can be done completely by the robot; it does not involve any kind of radiation exposure for the surgeon; with the knowledge of the characteristics of the stent grafts and the radiology we can chose the appropriate equipment and predict the results without being affected by the shape changing characteristics of the organs; its precision is better than that of a human; it is a low cost robot.

The paper is divided into several parts. Section 2 deals with abdominal aortic aneurysm. Section 3 is dedicated to a presentation of the a new prototype of robotic platform for computer-aided surgery. The new platform is composed of different components: a robotic system, an aorta phantom, a haptic device, and a real-time numerical simulation of an operation. Section 4 shows the experimental results of the 
whole system. Finally, a conclusion and some perspectives are provided.

\section{ENDOVASCULAR ANEURYSM REPAIR}

An Abdominal Aortic Aneurysm (AAA) (Figure 1) happens when the aorta gets dilated, to exceed more than fifty percent its initial diameter.

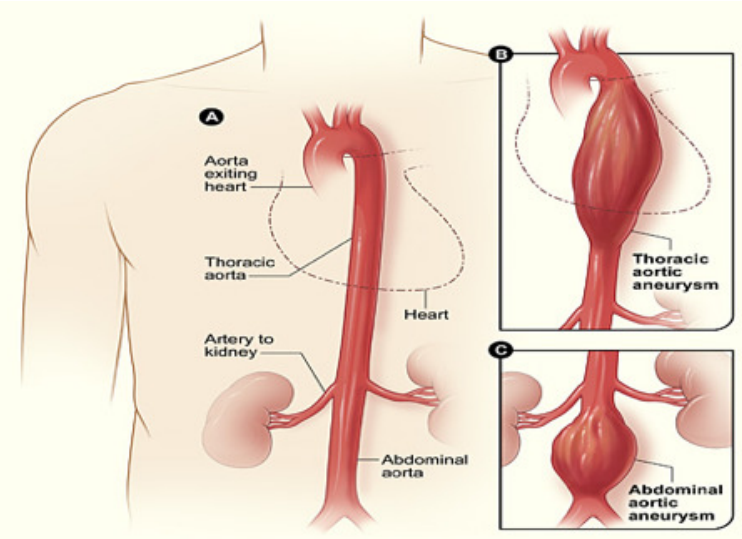

Fig. 1. Illustration of the Abdominal Aortic Aneurysm (National Institutes of Health, 2010)

This can lead to an explosion of the aorta causing the patient's death. In France, 5000 ruptures are experienced each year and the AAA is the $12^{\text {th }}$ leading cause of death. There are two ways to operate this disease: either by an open surgery or by an endovascular procedure. The difference of the procedures is that the open surgery takes from four to six hours and the recovery time is from five to ten days meanwhile the endovascular surgery takes from two to three hours and the recovery time is from two to three days. In other words, Endovascular Aneurysm Repair is not only minimally invasive but it is also faster and needs less recovery time than open surgery. The endovascular procedure is as follows: First the femoral artery has to be identifying, then, a catheter is inserted in that artery. Second, the catheter is guided to the aneurysm carrying a stent graft. Finally, the surgeon deploys it into place, once the stent graft is in position he/she removes the catheter (Figure 2 ) [1].

However, it also presents complications such as endoleak and stent-graft migration [4]. Endoleak happens when the stent graft does not seal well with the vessel wall; as a result, a bad outcome is obtained. In a study made by the division of vascular surgery of the Stanford University it is found that low initial deployment of the device, below the renal arteries, and short proximal fixation length are the principal causes of stent-graft migration. It is important to know that as we insert the guide and the stent-graft the shape of the aorta changes. The next section presents a new robotic platform dedicated for EVAR.

\section{MATERIAL AND METHODS}

\section{A. Prototype}

The proposed robot has to ensure two motions: The displacement of the guide wire along the $\vec{z}$ axis and the rotation

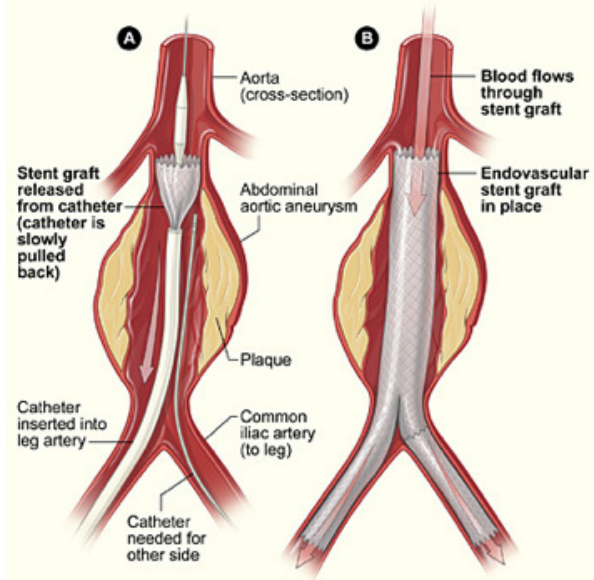

Fig. 2. Illustration of a stent graft placement (National Institutes of Health, 2011)

around the same axis. A kinematic scheme is provided on Figure 3.

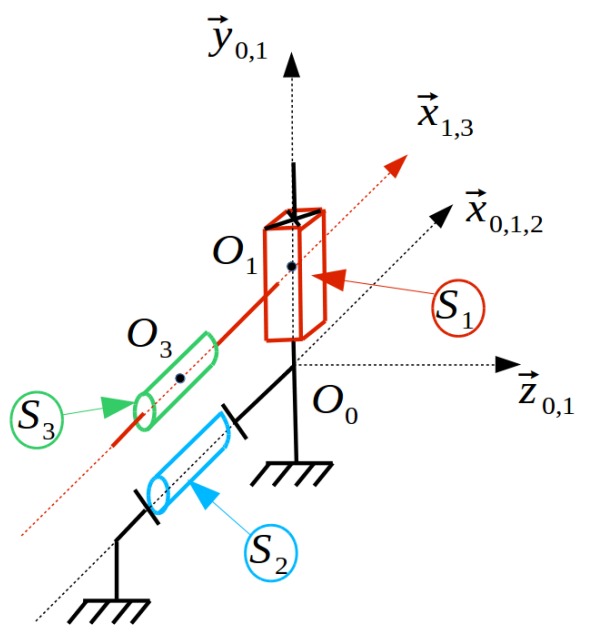

Fig. 3. Kinematic scheme of our robot

$\mathrm{S}_{2}$ and $\mathrm{S}_{3}$ are two rollers which let the guide wire to pass between them. Each roller is driven by a DC motor to ensure their rotations around the $\vec{x}_{1}$ axis. These motions will allow the pinched guide wire to translate along the $\vec{z}_{0,1}$ axis. As the link between $S_{1}$ and $S_{2}$ is a cylindrical joint, a translation of $S_{2}$ over $S_{3}$ will lead to rotate the pinched guide wire around the $\vec{z}_{0,1}$ axis. Finally, the link between $S_{1}$ and $S_{0}$ is a prismatic joint allowing the translation along the $\vec{y}_{0,1}$ axis. Thus the distance between $S_{2}$ and $S_{3}$ can be modified in order to use guide wires with different diameters. It is also possible to drive a catheter by this system instead of a guide wire.

A photo of our robot is shown on Figure 4. It consists of two conveyors placed one over the other which correspond to $S_{2}$ and $S_{3}$ on the kinematic scheme (Figure 3). The velocity of the conveyors is modified in order to control the position of the guide wire. 


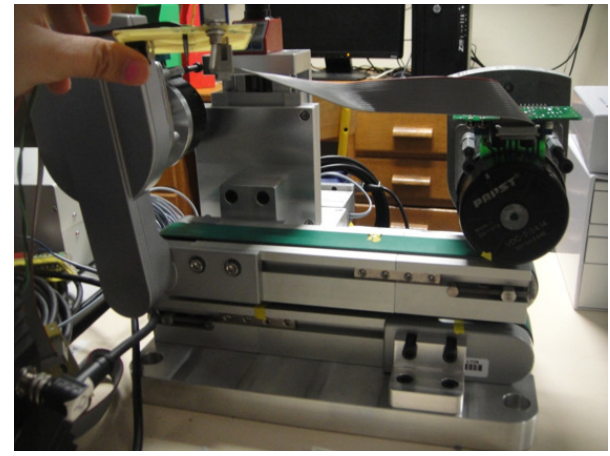

Fig. 4. Two conveyors that provide the displacement of the guide wire

The robotic platform also has a X-Y table which will be able to control the displacement of one conveyor over the other one, providing a rotation degree of freedom to the guide (Figure 5). This rotation will let the guide pass through some curvatures of the aorta.

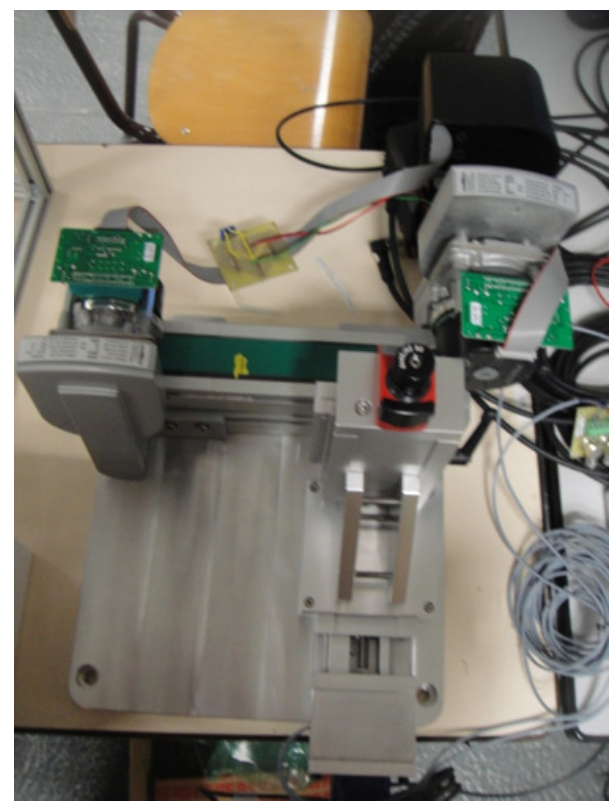

Fig. 5. $\mathrm{X}-\mathrm{Y}$ table to ensure the rotation of the guide wire

A phantom of the abdominal aorta and iliac arteries of a patient was printed by additive manufacturing in transparent silicone. Silicone can best represent arterial compliance during manipulations including a catheterization time (Figure 6). This component is held by springs, modeling the environment surrounding the aortic tree in the human body. This phantom is placed in a tank filled with water to allow the displacement of a hydrophilic flexible guide inside, by two entrance spots sealingly attached to the wall of the tank. This makes it possible to compare the actual insertion of the guide in the replica with the numerical model described in Section III.B.

Finally, in order to manipulate the robot it is necessary to have the control that best fits our requirements. We

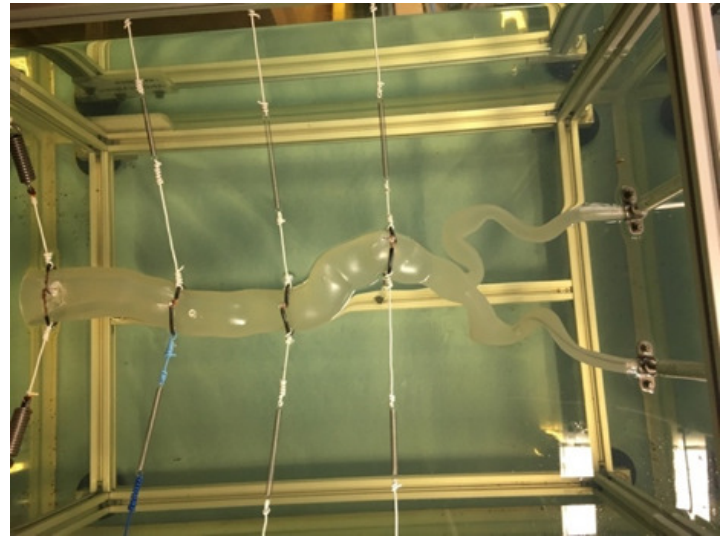

Fig. 6. Aorta phantom immersed in a tank of water

have to take in to account the price and specifications of different models and controls. A haptic device developed by Geomagic (Geomagic, Morrisville, North Carolina USA, 2013) was chosen (Figure 7). This device was chosen since we can achieve a good precision and it allows to perform the same movements as those performed by the surgeon during a conventional procedure. This device has six degrees of freedom of which three are motorized. It is also possible to collect the values of the six angles of rotation of the system including three gimbal angles.

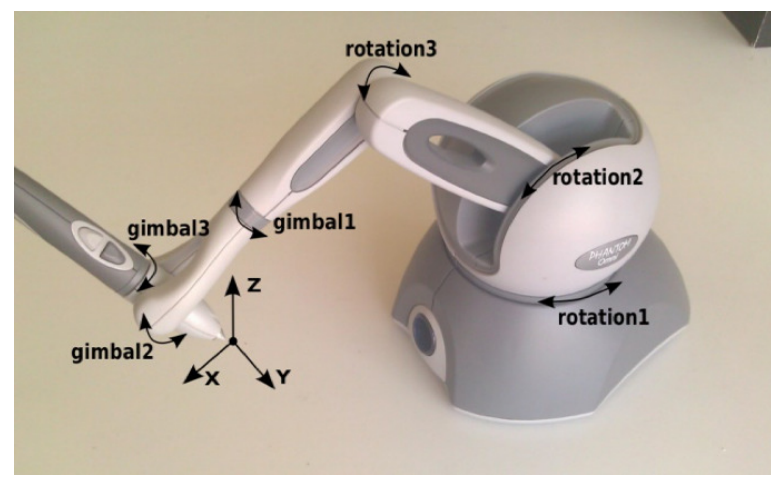

Fig. 7. Haptic device (Geomagic, 2013)

We can therefore describe different shapes in the defined workspace by holding the stylus of the haptic device. The latter is also equipped with two buttons that can be used for programming. Connected in USB to the computer, this hapic device allows the sending of a position reference to the conveyors and the X-Y table.

The phantom gives the sensation of contact. This is done since it is a motorized device that applies a force feedback on the users hand, allowing him/her to feel virtual objects and producing touch sensations. As a result, a realistic sense of touch is produced.

\section{B. Numerical Simulation Of An EVAR}

Another aspect of our work consists in combining the physical prototype described in section III.A and a numercial 
simulation of the movement of the guide in the aorta [5]. This work required the creation of a 3D digital model of the aorta and iliac arteries of a patient from medical imaging. The simplified whole structure of the aorta was reconstructed with the software ABAQUS and modeled with elastic, hyperelastic and linear composite materials. The mesh finite elements of the structure were created and several simulations were conducted. The model could be verified and optimized by superimposing medical images with the results of the simulation. The guide is inserted at a constant speed and angle and the model calculates the dynamic deformation of the aorta at the passage of the guide as well as the stress distribution in the structure. More details are available in [6]. Figure 8 shows the deformations caused by the insertion of different guides as well as the stress distribution in the aorta. The importance of the deformations makes it possible to understand the difficulty of the operation and the need for visual feedback during the procedure.

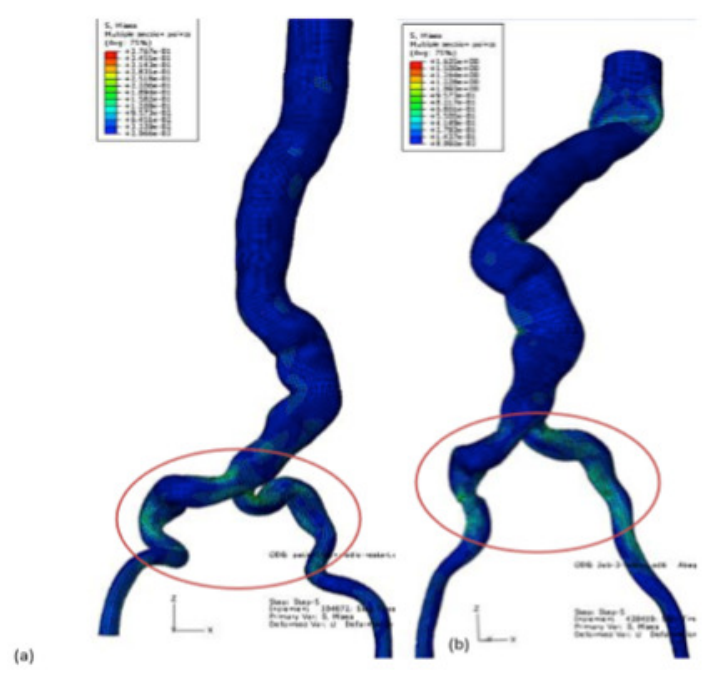

Fig. 8. Deformation and distribution of stress in the blood vessels under the action of the guides [6]

For our project, this simulation makes it possible to obtain information on the contacts between the guide and the aorta, their respective deformations and the trajectory of the guide. It also allows the trajectory planning of the operation and provides visual support for the practitioner. These numerical simulation will be essential in order to reproduce a realistic haptic feedback. The overall system includes all of the components described above. Figure 9 shows a picture of the overall system.

\section{Methods}

The essential aim is to reproduce as faithfully as possible the usual procedure, during which, the surgeon uses a guiding tool (Figure 10) that it serves around the guide to transmit a translation and / or a rotation through a force $\mathrm{F}$ and a moment M. For this, the Geomagic Touch haptic device is ideal with its stylus which has a geometry close to the original tool and which allows the same movements as the classical procedure.

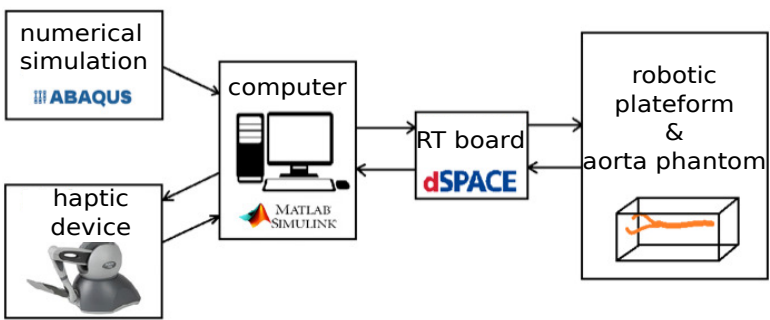

Fig. 9. Overall system

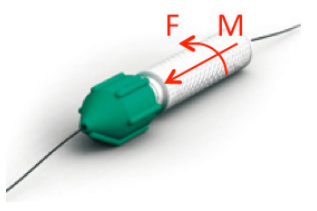

Fig. 10. Torque device

By pressing one of the button on the stylus of the haptic device as if it were pinching the guide, the surgeon will be able to transmit the prismatic movement or the rotation of the stylus to the robot. When the button is released, the stylus movements will no longer be recorded. In order to transmit the movements, the robot control can be separated into two parts: on one side the recovery of the data provided by the haptic device and their transformation into an usable signal, on the other hand, their use as a reference trajectory for the actuators of the robot.

The method used is therefore based on a master-slave system, where the haptic device control the robotic platform. For that, we used the results of the numerical simulation which include the efforts of contact between the end of the guide and the aortic wall as well as the length of guide inserted in the aorta at any moment. At the level of the robotic platform, this data is known thanks to measurement of the conveyors' speed and the insertion time. The approach of contact detection thus consists in comparing the length of guide actually inserted with the length of guide inserted in the simulation in order to find the corresponding contact in the simulation. This contact is finally returned to the haptic device in the form of a force whose the direction is opposite to the prismatic movement.

\section{RESUlTS}

\section{A. Trajectory tracking}

A program allowing the recovery of the reference position in translation from the haptic interface has been created. Figure 11 shows the juxtaposition of the stylus displacement curve and the switching curve of one of its buttons. The latter has been multiplied by 100 to allow a better reading. It has no unit for our application but corresponds to a square signal of amplitude $1 \mathrm{~V}$.

When one of the buttons of the haptic device is pressed for the first time, the coordinate $n_{0}$ of the stylus on the $\vec{z}$ 


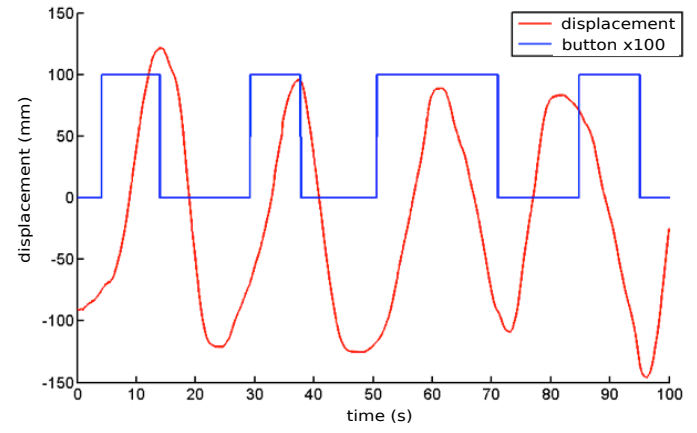

Fig. 11. Stylus displacement curve during an acquisition trajectory (red) and switch-on curve of button of the haptic device (blue)

axis (Figure 7) is saved and is subtracted from all the values that will be recorded during the movement (see Figure 12).

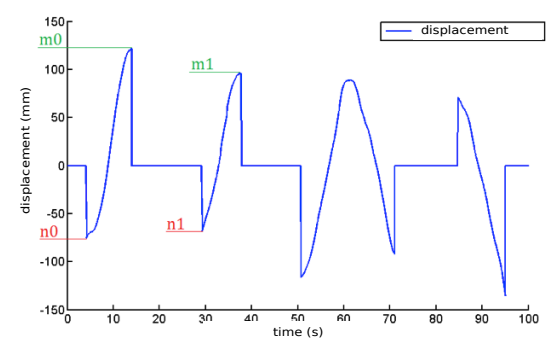

Fig. 12. Recorded stylus displacement curve for the creation of a reference trajectory

This is a correction of the signal to record the movement of the stylus from a zero value. When the button is released, meaning that the surgeon no longer wishes to transmit a movement to the guide, the last recorded value $m_{0}$ is maintained to allow the surgeon to start again from the point where it stopped as in the conventional procedure when the surgeon must move back the guide tool without changing the position of the guide. Once the button is pressed again, a new correction has be taken into account: it is therefore necessary both to cancel the first value $n_{1}$ and start from the last value $m_{0}$. This is repeated for each movement of the surgeon performed with the stylus following the engagement of the button. Figure 12 shows the curve obtained when multiplying the curves of Figure 11 between them. Only the movement made when the button is engaged is saved. This move will then be sent as a deposit to the robotic platform.

Finally, Figure 13 shows the position reference trajectory in its final form, obtained after data processing. The different movements of the stylus when the button 1 was engaged were accumulated to obtain this curve.

A real-time program using Simulink and ControlDesk softwares was developed. It uses the data collected through the haptic device and transmits it to the robotic platform through a proportional control law. It consists of three main parts: The first allows to send a voltage $(0 \mathrm{~V}$ or $5 \mathrm{~V})$ governing

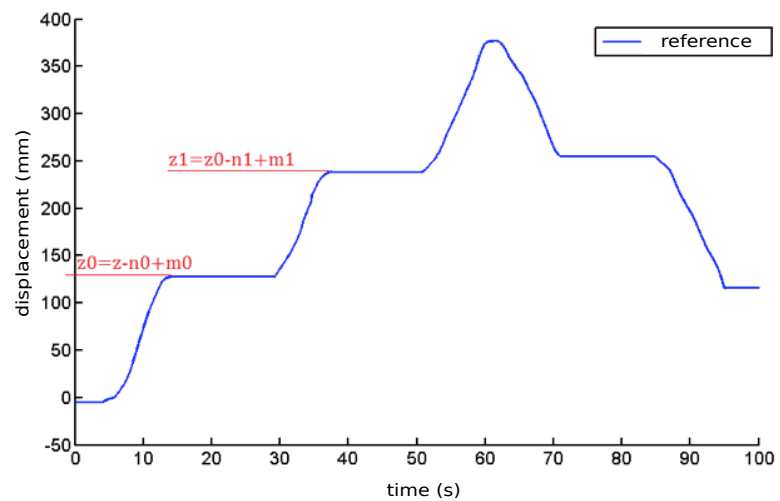

Fig. 13. Displacement of the stylus along the z-axis as a function of time after data processing

the direction of rotation of the conveyors through the sign of the derivative of the signal: the conveyors being coupled together, their directions of rotation will be opposed at any time. The second part sends a similar voltage to the two conveyors corresponding to the speed of the motors. This is between $0 \mathrm{~V}$ and $10 \mathrm{~V}$. Finally, the third part makes it possible to collect the value of the speed of the motors in the form of tension and to apply a correction to the signal sent. The following graph (Figure 14) presents the results obtained via ControlDesk from the data of the previous curve. The error between the two curves has been multiplied by ten to allow better visibility.

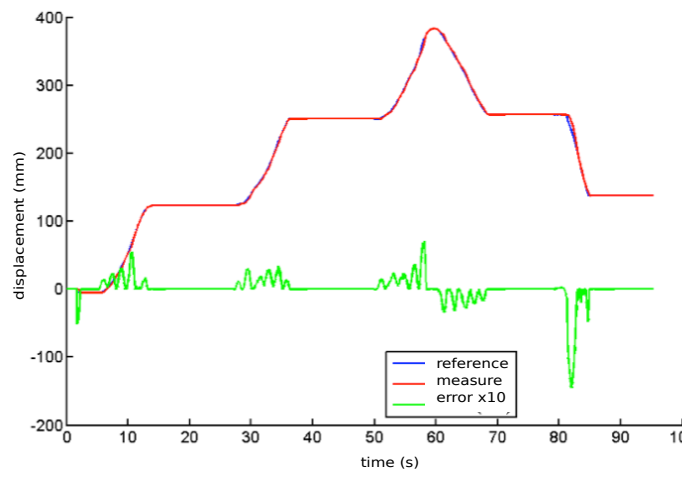

Fig. 14. Displacement of the guide as a function of time and tracking error curve

Similar to the prismatic movement of the guide, a program to collect the rotation of the stylus was written with MatlabSimulink. It works on the same principle as the previous one and makes it possible to obtain a curve similar to those of Figure 13, with this time an angle value.

\section{B. Haptic feedback}

The numerical model programmed with Abaqus provides data for the haptic feedback. For the latter, the only reliable information we have at the robotic platform is the guide length inserted into the aorta. In the model, the speed of insertion of the guide being constant and equal to $20 \mathrm{~mm} / \mathrm{s}$, 
the calculation of the guide length inserted is therefore to multiply the simulation time by this speed. Then, the Abaqus software allows to obtain the value of the contact forces exerted between the vascular wall and the end of the guide at each time step.

A Simulink program that combines rotation, translation movement and haptic feedback has been created. It retrieves data from previous curves and places them in a lookup table that returns the value of the corresponding contact force to the inserted guide length. Then, the inserted guide length is calculated in real time by multiplying the insertion speed by the time during which the motors of the conveyors operate thanks to the program managing the translation. This value is then sent to the look-up table so that finally the contact force is sent back to the haptic device.

\section{CONCLUSIONS}

Cardiovascular disease causes more deaths every year. Cases of abdominal aortic aneurysm rupture are no exception and physicians are increasingly resorting to endovascular surgery. This operation allows a faster recovery but remains risky: the slightest error can be fatal. To help surgeons during the procedure, a new technique has emerged: computerassisted surgery. In order to improve the existing techniques and secure the operation, we presented in this paper a remote control system of the procedure. The project is divided into two parts: a numerical study that involves modeling the insertion of the guide virtually and an automatic study that consists in managing the control of the insertion of the guide via a robotic platform. The current manuscript is focused on the second part of the study. Its main purpose is to present the different components of a new robotic platform and to implement a control law to a haptic device. This device allows both the control of insertion of the guide and the haptic feedback of the contact between the guide and the wall to allow the surgeon to perform the procedure remotely while keeping the same sensations as in a manual procedure. The work was divided into several phases. First of all, the design of a robotic platform, then the programming of the various functions allowing the retrieval of the data related to the movement of the haptic device. Then, these data were processed to allow the control of the robotic platform. Due to a compatibility problem between the interfaces (the MATLAB / Simulink software, the haptic interface and the dSPACE acquisition system), these two steps were performed separately. Despite the encouraging results, the real-time management of the platform was not possible so far. The haptic feedback has also been implemented in the system by importing the contact data from the numerical simulation. By means of a correspondence table, the appropriate force is returned to the haptic interface as a function of the length of the inserted guide. However, this force does not correspond to the force felt at the tool but to the force acting on the guide in contact with the wall. Finally, these different programs have been coupled to create a control interface. This one presents a haptic feedback without tremors, a management of the recoil and also an exit of the contact for an arbitrary rotation of $90^{\circ}$.
In order to enable real-time management of the platform, a Microsoft Visual Studio application has been created. Based on the study of demonstration programs, it allowed to get rid of MATLAB / Simulink software, bypassing the problem of cross-compilation. The application, written in $\mathrm{C}$ language, interacts with the haptic device and provides access to the acquisition card and therefore with the robotic platform. We have been able to reprogram the control of the platform (translation, rotation) as well as the haptic feedback thanks to the data of the numerical simulation. In the continuation of the work, the most urgent will be to improve this application by optimizing these features. At the level of the haptic feedback, the measurement of the forces at the level of the guiding tool must be carried out in order to return suitable force values. The program should also be tested on several simulations to verify its robustness.

\section{ACKNOWLEDGMENT}

The authors would like to express my very great appreciation to Indira Gomez Osorio and Romain Rémy for their contributions to this work. The authors would also like to thank Dr. Vincenzo Brizzi and Pr. Patrick Lermusiaux for their valuable and constructive suggestions during the planning and development of this research project. Their willingness to give their time so generously have been very much appreciated.

\section{REFERENCES}

[1] S. Amiot, S. Haulon, J.-P. Becquemin, P.-E. Magnan, P. Lermusiaux, Y. Goueffic, E. Jean-Baptiste, F. Cochennec, J.-P. Favre, and Association Universitaire de Recherche en Chirurgie Vasculaire. Fenestrated endovascular grafting: The french multicentre experience. European journal of vascular and endovascular surgery : the official journal of the European Society for Vascular Surgery, 39:537-544, May 2010.

[2] United Kingdom E. V. A. R. Trial Investigators, Roger M. Greenhalgh, Louise C. Brown, Janet T. Powell, Simon G. Thompson, David Epstein, and Mark J. Sculpher. Endovascular versus open repair of abdominal aortic aneurysm. The New England journal of medicine, 362:18631871, May 2010.

[3] Karon E. MacLean. Haptic interaction design for everyday interfaces. Reviews of Human Factors and Ergonomics, 4(1):149-194, 2008.

[4] Geert Maleux, Marcel Koolen, and Sam Heye. Complications after endovascular aneurysm repair. In Seminars in interventional radiology, volume 26, pages 003-009. (C) Thieme Medical Publishers, 2009.

[5] Ghizlane Mouktadiri. Angiovision - Pose d'endoprothese aortique par angionavigation augmentée. PhD thesis, INSA Lyon, 2012.

[6] Ghizlane Mouktadiri, Benyebka Bou-Said, and Helene Walter-Le-Berre. Aortic endovascular repair modeling using the finite element method. Journal of Biomedical Science and Engineering, 6:917-927, 2013

[7] Adnan I. Qureshi, Hossam Egila, Malik M. Adil, Harris Siddiqi, Nidaullah Mian, Ameer E. Hassan, Jefferson T. Miley, Gustavo J. Rodriguez, and M. Fareed K. Suri. "no turn back approach" to reduce treatment time for endovascular treatment of acute ischemic stroke. Journal of stroke and cerebrovascular diseases : the official journal of National Stroke Association, 23:e317-e323, 2014.

[8] Junchen Wang, Takashi Ohya, Hongen Liao, Ichiro Sakuma, Tianmiao Wang, Iwai Tohnai, and Toshinori Iwai. Intravascular catheter navigation using path planning and virtual visual feedback for oral cancer treatment. The international journal of medical robotics + computer assisted surgery : MRCAS, 7:214-224, June 2011.

[9] Tianmiao Wang, Dapeng Zhang, and Liu Da. Remote-controlled vascular interventional surgery robot. The international journal of medical robotics + computer assisted surgery : MRCAS, 6:194-201, June 2010. 